\title{
Full Activation of Boron in Silicon Doped by Self-Assembled Molecular Monolayers
}

\author{
Xuejiao Gao, ${ }^{\dagger, \mathbb{I}}$ Ilia Kolevatov, ${ }^{\ddagger}$ Kaixiang Chen, ${ }^{\dagger}$ Bin Guan, ${ }^{\dagger, \#}$ Abdelmadjid Mesli, ${ }^{\S}$ Edouard Monakhov, \\ and Yaping Dan*, ${ }^{\dagger}$ \\ ${ }^{\dagger}$ University of Michigan - Shanghai Jiao Tong University Joint Institute, Shanghai Jiao Tong University, Shanghai 200240, China \\ ${ }^{\ddagger}$ Department of Physics, Center for Material Science and Nanotechnology, University of Oslo, Blindern, P.O. Box 1048, N-0316 \\ Oslo, Norway \\ ${ }^{\S}$ Institut Matériaux Microélectronique Nanosciences de Provence, UMR 6242 CNRS, Université Aix-Marseille, 13397 Marseille \\ Cedex 20, France
}

Supporting Information

ABSTRACT: Self-assembled molecular monolayer (SAMM) doping has great potential in state-of-the-art nanoelectronics with unique features of atomically precision and nondestructive doping on complex $3 \mathrm{D}$ surfaces. However, it was recently found that carbon impurities introduced by the SAMM significantly reduced the activation rate of phosphorus dopants by forming majority carrier traps. Developing a defect-free SAMM-doping technique with a high activation rate for dopants becomes critical for reliable applications. Considering that susbstitutional boron does not interact with carbon in silicon, herein we employ Hall measurements and secondary ion mass spectrometry (SIMS) to investigate the boron activation rate and then deep level transient spectroscopy (DLTS) and minority carrier transient spectroscopy (MCTS) to analyze defects in boron-doped silicon by the SAMM technique. Unlike the phosphorus dopants, the activation rate of boron dopants is close to $100 \%$, which is consistent with the defect measurement results (DLTS and MCTS). Only less than 1\% boron dopants bind with oxygen impurities, forming majority hole traps. Interestingly, carbon-related defects in the form of $\mathrm{C}_{s} \mathrm{H}$ and $\mathrm{C}_{s} \mathrm{OH}$ act as minority trap states in boron-doped silicon, which will only capture electrons. As a result, the high concentration of carbon impurities has no impact on the activation rate of boron dopants.

KEYWORDS: full activation, boron-doped silicon, molecular monolayer doping, carbon-related defects, minority carrier trap

\section{INTRODUCTION}

Self-assembled molecular monolayer (SAMM) doping enables atomically precise and damage-free doping on $3 \mathrm{D}$ surfaces and thus may find important applications in developing deep nanometer-scale electronics. ${ }^{1-4}$ In this doping process, dopantcontaining molecular monolayers are first covalently bonded onto hydrogen-terminated semiconductor surfaces. A thermal process is then employed to drive the dopants into the semiconductor bulk, and the dopants are electrically activated at the same time. ${ }^{5}$ Ruess et al. first demonstrated an ultrahigh vacuum monolayer doping technique in $2004^{6}$ and later adopted scanning tunneling microscopy (STM) to covalently graft single phosphine molecules onto a silicon surface followed by incorporating annealing to activate phosphorus. ${ }^{7,8}$ For large-scale applications, a solution-based monolayer doping was demonstrated ${ }^{1}$ in 2008 where thermal energy ${ }^{1}$ or ultraviolet irradiation ${ }^{9}$ was employed to generate surface radicals for covalent attachment of doping molecules. Up to now, this doping technology has been applied to sub-5 nm ultrashallow junction formation, ${ }^{10}$ conformal doping on fin-

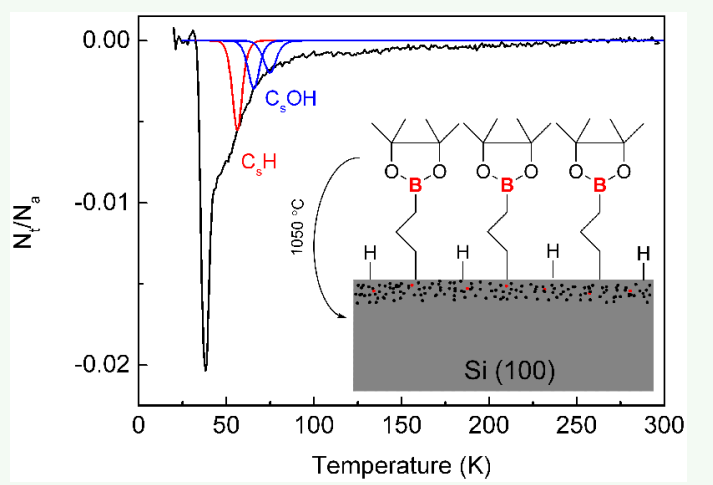

FETs, ${ }^{11}$ and atomic-scale surface patterning. ${ }^{12,13}$ In addition, single dopants may be potentially controlled at large scale by combining "top-down" large-scale nanofabrication and "bottom-up" self-assembly. ${ }^{14}$

One of the main concerns for solution-based SAMM doping is organic contamination and dopant deactivation. ${ }^{15-17}$ Shimizu et al. ${ }^{16}$ have demonstrated that carbon and oxygen diffused unintentionally into silicon during the thermal annealing process, and they suggested to sacrifice a top layer of 2-3 nm for industrial application. Recently, we found that carbon contaminants from organic molecules can electrically deactivate at least $20 \%$ of the phosphorus. ${ }^{15,18}$ Taheri et al. proposed gas-phase molecule doping, which not only removed the organic solvent but also increased the uniformity and controllability. ${ }^{19}$ Holmes and co-workers grew a thin $\mathrm{SiO}_{2}$ layer to achieve a minimal carbon contamination. ${ }^{20}$ However,

Received: November 8, 2019

Accepted: December 10, 2019

Published: December 10, 2019 
all these investigations were on phosphorus-doped silicon. Little attention was paid to how the carbon or oxygen in organic carriers interact with boron dopants in silicon and whether electrically active defects exist in boron-doped silicon via SAMM.

In this work, we investigated boron-doped silicon by SAMM from the aspects of activation rate and defects. Allylboronic acid pinacol ester (ABAPE) molecules were applied to introduce boron dopants into silicon by SAMM doping. The hole and boron dopant concentrations were measured by Hall effect measurement and secondary ion mass spectrometry (SIMS), respectively. To fully understand the defects in SAMM-doped silicon, we employed deep level transient spectroscopy (DLTS) and minority carrier transient spectroscopy (MCTS) to investigate the majority and minority carrier trap states, respectively. The results show that carbon impurities do not bind with boron dopants. Carbon impurities only form minority carrier trap states that have no impact on the ionization rate of boron dopants, as a result of which boron dopants are close to full activation. Only oxygen impurities will bond with boron dopants, resulting in the deactivation of less than $1 \%$ boron dopants.

\section{RESULTS AND DISCUSSIONS}

ABAPE monolayers were covalently bonded onto a silicon surface following the process described in refs 1 and 15. A compact and robust molecular monolayer was demonstrated by comparing X-ray photoelectron spectroscopic (XPS) spectra of blank sample (freshly HF-etched $\mathrm{Si}$ ), control sample $(\mathrm{ABAPE} / p$-xylene $=0: 1)$, and ABAPE-functionalized sample $(\mathrm{ABAPE} / p$-xylene $=1: 2)$, as shown in Figure 1 and Supplementary Figures S2,S3. In Figure 1a, the silicon dioxide peak $(102.9 \mathrm{eV})$ is nearly invisible on the freshly HF-etched silicon surface, and a $\mathrm{Si} 2 \mathrm{p}$ peak at $99.5 \mathrm{eV}$ due to the $\mathrm{Si}$ substrate splits into $\mathrm{Si} 2 \mathrm{p}(3 / 2)$ at $99.2 \mathrm{eV}$ and $\mathrm{Si} 2 \mathrm{p}(1 / 2)$ at $99.8 \mathrm{eV}$. After heat treatment in $p$-xylene, the silicon dioxide peak at $102.9 \mathrm{eV}$ (fwhm $1.8 \mathrm{eV}$ ) shows up on the control sample due to surface oxidation (Figure 1c). However, with a compact ABAPE monolayer covering the silicon surface, only a tiny silicon dioxide peak was detectable on the B-functionalized silicon surface (Figure 1e), indicating that a compact and robust monolayer of ABAPE has been successfully grafted. No B 1s was detected on blank sample (Figure 1b) and control sample (Figure 1d), but a broad peak of Si 2s plasma loss was detected. In contrast, after functionalization with ABAPE, a peak at $191.3 \mathrm{eV}$ (fwhm $1.5 \mathrm{eV}$ ) was present in Figure 1f, which is ascribed to the boron in the ABAPE monolayer. In addition, $C$ 1s narrow scans also support the successful coupling of boron-containing molecules, as shown in Supplementary Figure S3.

To evaluate the boron doping process, SAMM doping was first performed on a high-resistivity silicon wafer $(>10 \mathrm{k} \Omega \mathrm{cm})$. Experiment details can be found in our previous publication. ${ }^{15}$ A control sample was prepared by removing ABAPE in $p$ xylene solvent. van der Pauw four-probe measurements were applied in darkness to measure the sheet resistance. Table 1 compares the experiment results of blank sample (bare silicon wafer), control sample, and B-doped sample. The sheet resistance of the control sample $(299.60 \mathrm{k} \Omega / \square)$ is very close to that of blank sample $(315.82 \mathrm{k} \Omega / \square)$, which indicates that SAMM doping introduces negligible contamination. After boron was doped into silicon via SAMM, the sheet resistance dramatically decreased to $3.20 \mathrm{k} \Omega / \square$. This drop in sheet
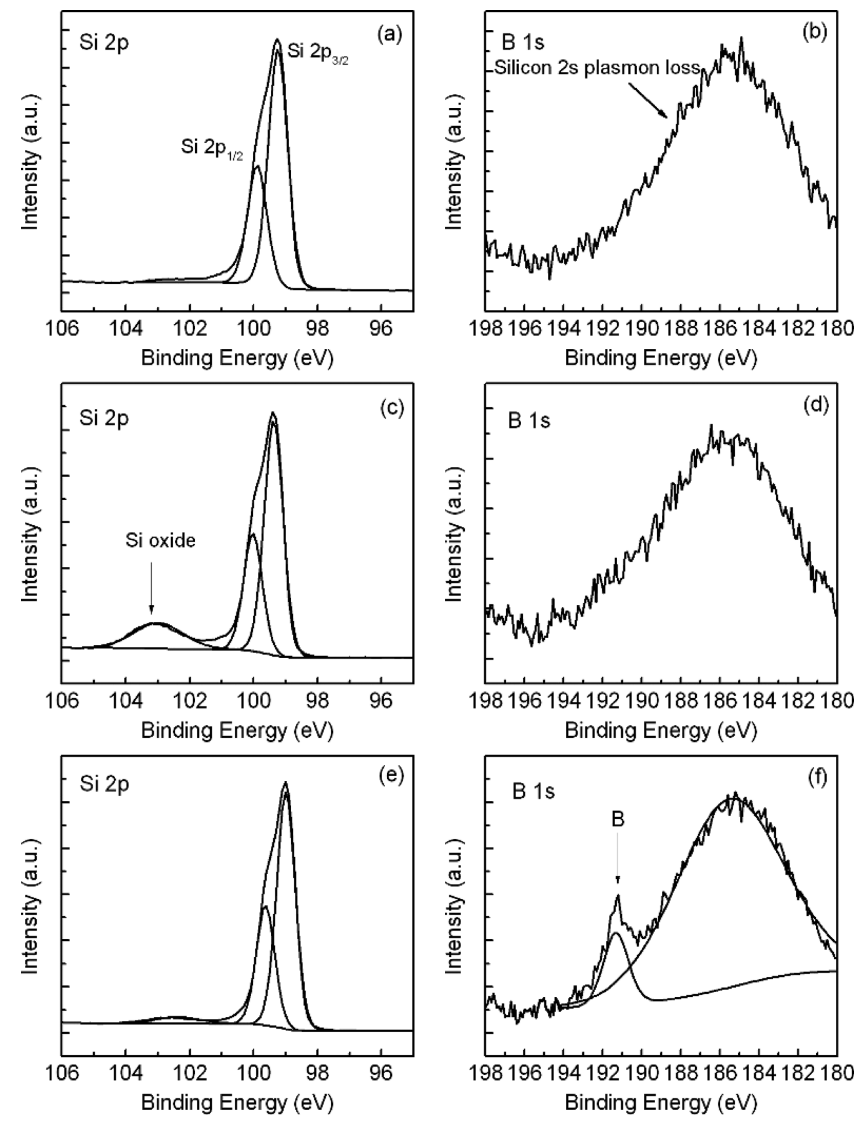

Figure 1. XPS narrow scans of blank sample, control sample, and ABAPE-functionalized sample. (a) and (b) are Si $2 p$ and B 1 s narrow scans on blank sample. (c) and (d) are Si $2 \mathrm{p}$ and B 1 s narrow scans on control sample. (e) and (f) are $\mathrm{Si} 2 \mathrm{p}$ and B 1 s narrow scans on ABAPE-functionalized sample. The takeoff angle is $45^{\circ}$.

Table 1. Sheet Resistance of Blank Sample, Control Sample, and Boron-Doped Samples by van der Pauw Technique

\begin{tabular}{cc} 
& sheet resistance $(\mathrm{k} \Omega / \square)$ \\
blank sample & 315.82 \\
control sample & 299.60 \\
boron-doped sample & 3.20 \\
\hline
\end{tabular}

resistance is exclusively attributed to molecular monolayer doping, and hence, effective boron doping in silicon has been demonstrated.

As we have discovered that carbon contamination from the organic dopant carriers can deactivate as least $20 \%$ of the phosphorus dopants in phosphorus-doped silicon by SAMM, ${ }^{15}$ the investigations on the activation rate and defects in borondoped silicon became the keynote of this research. First, the boron distribution near the silicon surface was analyzed by SIMS. As shown in Figure 2a, the boron concentration rapidly drops from $1.59 \times 10^{19}$ at the surface to $2.6 \times 10^{18} \mathrm{~cm}^{-3}$ at $\sim 5$ $\mathrm{nm}$ below the surface. The boron dopants then drop to $6.1 \times$ $10^{15} \mathrm{~cm}^{-3}$ at $\sim 104 \mathrm{~nm}$ below the surface. Because a monolayer of ABAPE is grafted on the silicon surface, the total amount of initial dopants is limited. The SIMS data from 5 to $104 \mathrm{~nm}$ agrees well with the limited-source diffusion. By fitting the limited-source diffusion model into SIMS data, ${ }^{9}$ a diffusivity of $7.24 \times 10^{-15} \mathrm{~cm}^{2} \mathrm{~s}^{-1}$ was obtained, comparable to the diffusivities for boron reported in the literature. ${ }^{21}$ The sharp decrease at the first $5 \mathrm{~nm}$ was likely related with the observed 

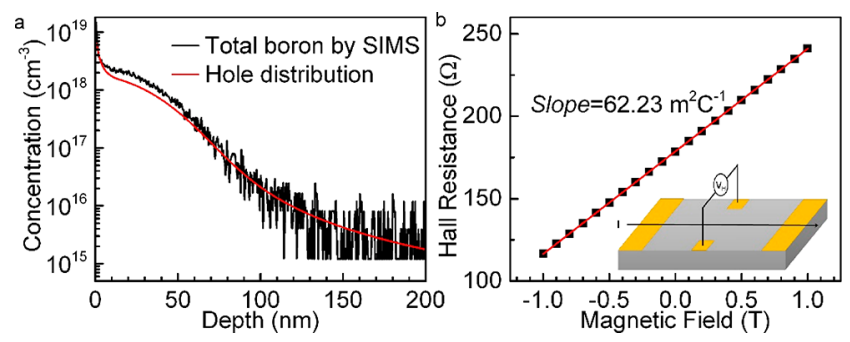

Figure 2. (a) Boron depth profile measured by quadruple SIMS (black curve). Red line is hole distribution simulated in Supplementary Note 3. (b) Hall resistance as a function of the perpendicular magnetic field.

drop of temperature in the tube furnace at the initial stage of thermal annealing. Integrated from 0 to $150 \mathrm{~nm}$, the surface boron concentration is $1.02 \times 10^{13} \mathrm{~cm}^{-2}$.

Note that when the boron concentration is higher than $1 \times$ $10^{17} \mathrm{~cm}^{-3}$, boron dopants will be partially ionized, and a maximum $25 \%$ incomplete ionization will be achieved at $\sim 5 \times$ $10^{18} \mathrm{~cm}^{-3} \cdot{ }^{22,23}$ In Supplementary Note 3, the hole distribution or active boron distribution was calculated in consideration of the incomplete ionization due to high concentration as well as the built-in internal electric field caused by nonuniform dopant distribution, if we assume that all boron dopants are electrically active. The red curve in Figure 2a shows the calculated hole distribution. After integration, the surface concentration of holes is $7.97 \times 10^{12} \mathrm{~cm}^{-2}$, meaning that only $78.14 \%$ boron dopants will create holes in the valence band due to incomplete ionization.

Then, Hall measurements were performed on the B-doped sample at room temperature with a Hall bar geometry (Figure $2 b$, inset). As shown in Figure $2 b$, with the magnetic field scanning from -1 to $1 \mathrm{~T}$, the Hall resistance has a linear correlation with magnetic field (black dots). After fitting (red line), a slope of $62.23 \mathrm{~m}^{2} \mathrm{C}^{1-}$ was obtained, and the hole concentration $p$ is calculated with eq 1 .

$$
p=\frac{r}{e R_{\mathrm{H}}}=\frac{r B}{e d\left(\Delta V_{\mathrm{H}} / I\right)}
$$

where $r$ is the Hall correction factor, $e$ is the unit charge, $R_{\mathrm{H}}$ is the Hall coefficient, $B$ is the magnetic field, $d$ is the doping depth, $\Delta V_{\mathrm{H}}$ is the Hall voltage, $I$ is the source current, and $\left(\Delta V_{\mathrm{H}} / I\right)$ is the Hall resistance.

Considering that the SAMM-doping region is nonuniform from surface to bulk, we calculate the surface hole concentration $N_{\mathrm{p}}$ with eq. 2 .

$$
N_{\mathrm{p}}=\int_{0}^{d} p(x) \mathrm{d}_{x}=\frac{r B}{e\left(\Delta V_{\mathrm{H}} / I\right)}=\frac{r}{e \times \text { slope }}
$$

Due to the scatterings by phonons, ionized impurities, and neutral impurities, a Hall correction factor of 0.76 is chosen, ${ }^{24}$ and the hole concentration is calculated to be $7.6 \times 10^{12} \mathrm{~cm}^{-2}$. We previously found from SIMS data that the hole concentration would be $7.97 \times 10^{12} \mathrm{~cm}^{-3}$ if we assume that all boron dopants are electrically active. The hole concentration obtained from the Hall measurements indicate that $\sim 95 \%\left(=7.6 \times 10^{12} / 7.97 \times 10^{12}\right)$ of all boron dopants are electrically active. In fact, the hole concentration found from Hall measurements is underestimated up to $10 \%$, because the boron distribution is highly nonuniform near surface. ${ }^{14}$ But at the same time, we have some uncertainties $(\sim 10 \%)$ in the empirical Hall correction factor and hole mobilities. What we can conclude is that the boron dopants in SAMM-doped silicon are close to the full activation. This conclusion is consistent with what we found from the following DLTS and MCTS measurements.

For DLTS measurements, Schottky diodes were fabricated on a SAMM-doped p-type silicon substrate with a resistivity of $10-20 \Omega \mathrm{cm}$. A background boron concentration of $6 \times 10^{14}$ $\sim 1 \times 10^{15} \mathrm{~cm}^{-3}$ is required to probe the SAMM-doped region near the surface and reduce the series resistance in Schottky diodes. ${ }^{15}$ Schottky and Ohmic contacts (Figure 3a) were fabricated by depositing $200 \mathrm{~nm}$ thick Ti on the front surface
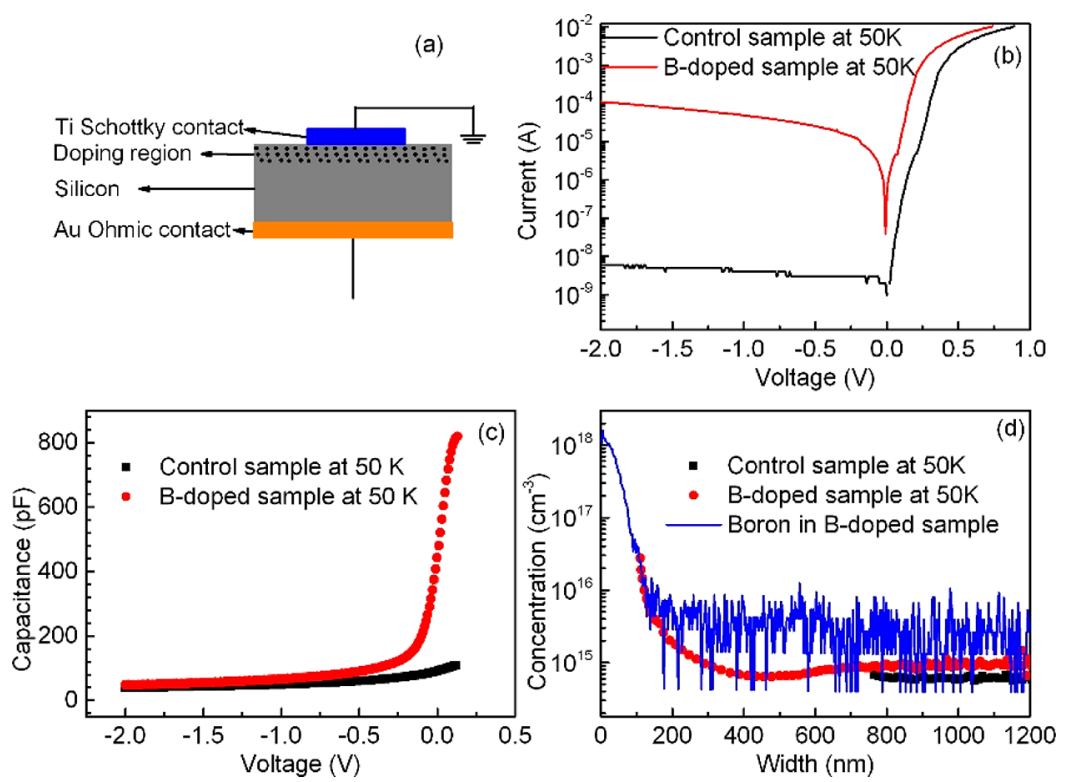

Figure 3. (a) Schematic of the Schottky diode. (b) $I-V$ curves of control sample and SAMM-doped sample at $50 \mathrm{~K}$. (c) $C-V$ curves of control sample and boron-doped sample at $50 \mathrm{~K}$. (d) Ionized charge profile of control sample (black dots) and boron-doped sample (red dots). The blue curve is the boron depth profile measured by SIMS 

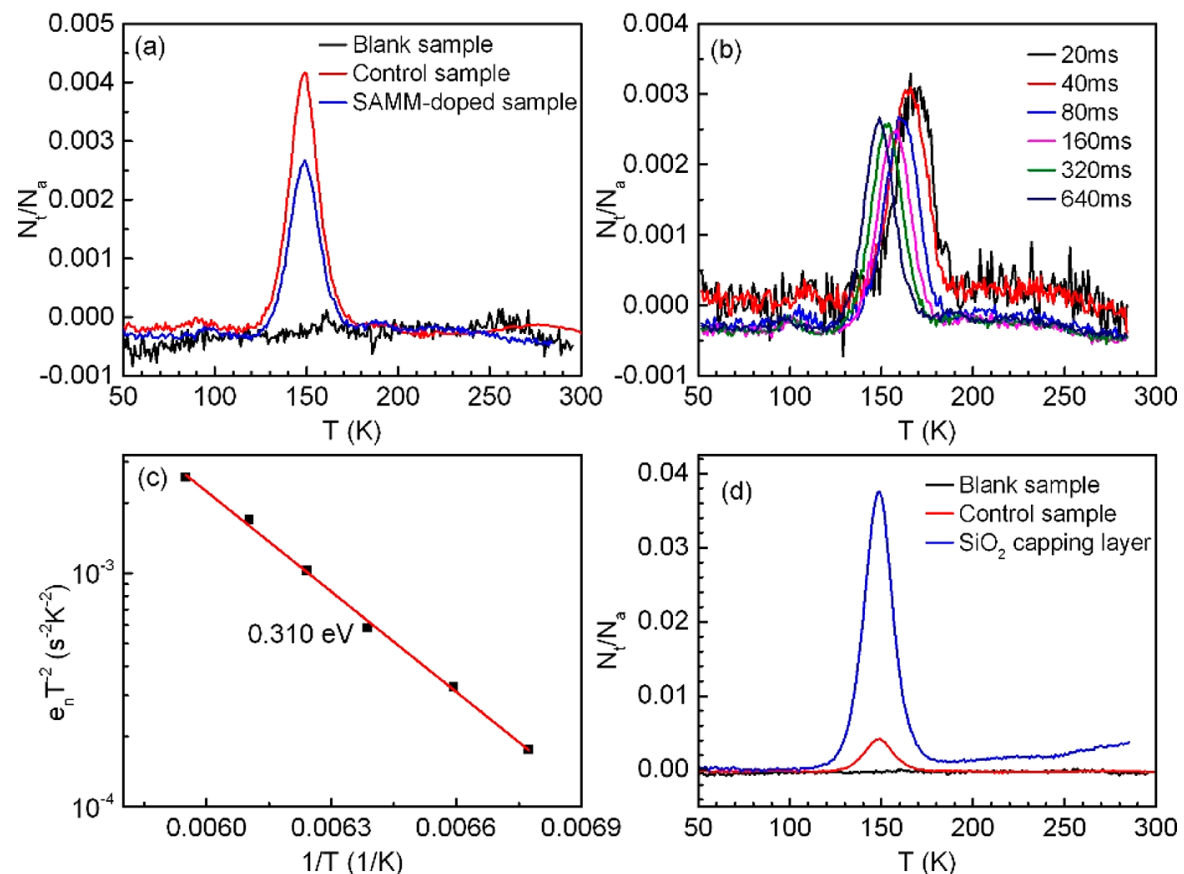

Figure 4. (a) DLTS measurements of blank sample (black line), control sample (red line), and boron-doped sample (blue line) with reversed biases of $-1.2,-1.25$, and $-2 \mathrm{~V}$, respectively. Pulse voltages are all set to be $0 \mathrm{~V}$, and the rate window is $640 \mathrm{~ms}^{-1}$. (b) DLTS spectra measured on the SAMM-doped sample with a rate window decreasing from 640 to $20 \mathrm{~ms}^{-1}$. (c) Arrhenius plot of SAMM-doped sample with peak positions (in $\mathrm{K}$ ) and rate windows (in $\mathrm{ms}^{-1}$ ) derived from b. (d) DLTS spectra of control sample (red line) compared with silicon annealing with $\mathrm{SiO}_{2}$ layer at $1050{ }^{\circ} \mathrm{C}$ for $2 \mathrm{~min}$.

a

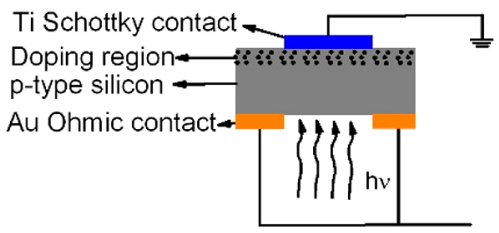

b

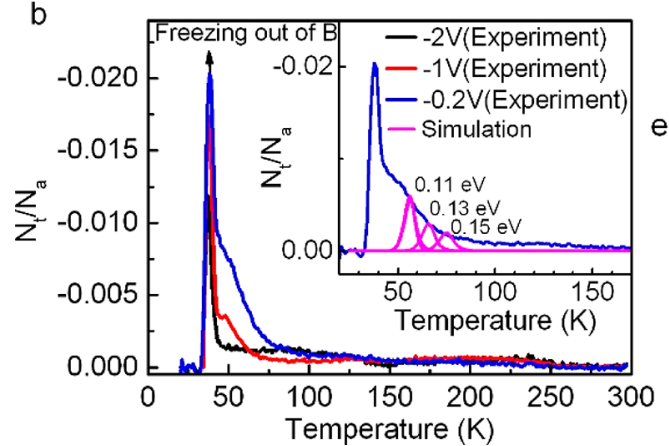

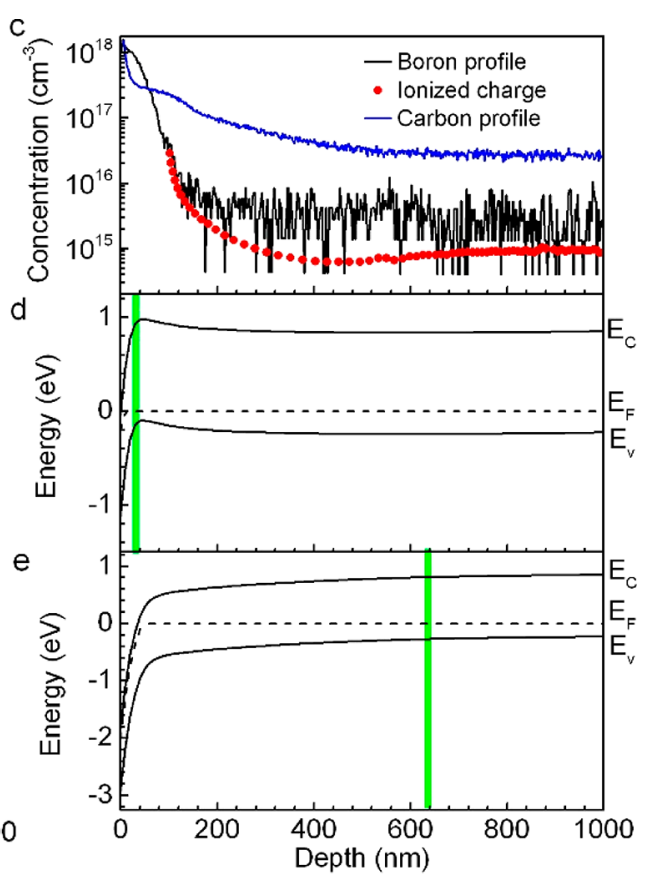

Figure 5. (a) Schematic of MCTS sample. Light with wavelength of $940 \mathrm{~nm}$ shines from the back side. (b) MCTS spectra of SAMM-doped sample with bias voltages of $-2 \mathrm{~V}$ (black line), $-1 \mathrm{~V}$ (red line), and $-0.2 \mathrm{~V}$ (blue line). Rate window is $640 \mathrm{~ms}^{-1}$. Inset: MCTS simulation with bias voltage of $-0.2 \mathrm{~V}$. (c) Carbon depth profile was measured by SIMS (blue line). As a reference, the boron profile (black line) and charge profile (red dots) are also present. (d) Simulated band structure and MCTS detection region at bias voltage of -0.2 V. (e) Simulated band structure and MCTS detection region at bias voltage of $-2 \mathrm{~V}$.

and $100 \mathrm{~nm}$ thick $\mathrm{Au}$ on the back surface. From currentvoltage $(I-V)$ curves in Figure $3 \mathrm{~b}$, Schottky junctions have been successfully formed on both the control and SAMMdoped samples. With a surface concentration as high as $2 \times$ $10^{18} \mathrm{~cm}^{-3}$ (Figure $3 \mathrm{~d}$ ), the built-in potential height is reduced, and therefore, four orders of magnitude enhancement of leakage current was measured on SAMM-doped sample. As the voltage increased from -2 to $0.2 \mathrm{~V}$ in Figure $3 \mathrm{c}$, the junction capacitance on the SAMM-doped sample rapidly increases after $-0.7 \mathrm{~V}$, which is attributed to the extraneous boron from 
the SAMM-doping process. The ionized charge profiles for the control (black) and SAMM-doped sample (red) were extracted from the $\mathrm{CV}$ measurements, which are consistent with the SIMS profile (blue) as shown in Figure 3d.

DLTS measurements were subsequently carried out with temperature scanning from 50 to $300 \mathrm{~K}$ to study the majority carrier traps (Figure 4a). Reversed biases for blank, control, and boron-doped sample were set to be $-1.2,-1.25$, and -2 $\mathrm{V}$. Pulse voltages were all set at $0 \mathrm{~V}$. The DLTS spectrum of blank sample remains constant near zero (black line), demonstrating that the concentration of defects in silicon substrate is negligibly small. However, a peak at $148.8 \mathrm{~K}$ appears unexpectedly on both the spectra of the control sample (red line) and boron-doped sample (blue line). In addition, the peak amplitude measured on the control sample is higher than that on the SAMM-doped sample. To get the energy level of corresponding defect, a series of spectra with rate windows decreasing from $640 \mathrm{~ms}^{-1}$ to $20 \mathrm{~ms}^{-1}$ were tested as shown in Figure $4 \mathrm{~b}$. With peak positions and corresponding rate windows, the Arrhenius plot was drawn in Figure 4c (black dots). From the slope of the linear fitting, a defect energy level of $0.310 \mathrm{eV}$ is extracted, which can be attributed to the following alternatives, $\mathrm{B}_{\mathrm{s}}-\mathrm{O}_{2 \mathrm{i}}$ (substitutional boron bonded with oxygen dimer) ${ }^{25,26}$ or $\mathrm{B}_{\mathrm{i}}-\mathrm{C}_{\mathrm{s}}$ (interstitial boron bonded with substitutional carbon). ${ }^{27,28}$ To reveal more information about this defect energy level and exclude the concerns that this peak may be from unintentional contamination, another control sample is carefully prepared with an e-beam evaporated $\mathrm{SiO}_{2}$ capping layer and annealed at the same condition. As the blue curve in Figure $4 \mathrm{~d}$ shows, the same peak at $148.8 \mathrm{~K}$ appears with an amplitude about 10 times higher than that on the control sample (red line in Figure 4d). Obviously, the appearance of the defects is related to the $\mathrm{SiO}_{2}$ capping layer. Hence, we ascribe this defect to be $\mathrm{B}_{\mathrm{s}}-\mathrm{O}_{2 \mathrm{i}}$ with oxygen diffusing from the $\mathrm{SiO}_{2}$ capping layer. ${ }^{29}$ The defect concentration is less than $1 \%$ of the boron concentration.

Previous studies have shown that a complex of substitutional carbon $\left(\mathrm{C}_{\mathrm{s}}\right)$ and hydrogen will form a defect level at $0.15 \mathrm{eV}$ below the conduction band minimum. ${ }^{30}$ With the influence from oxygen, two different complexes of $\mathrm{C}_{\mathrm{s}} \mathrm{OH}$ with energy levels of 0.11 and $0.13 \mathrm{eV}$ were observed. ${ }^{31,32}$ These defect states near the conduction band will not trap majority holes, and DLTS cannot detect these defects as we showed above. Here we use MCTS to investigate these carbon-related defects. As shown in Figure 5a, a window was opened at the back side of the Schottky diodes, and an optical pulse (at a wavelength of $940 \mathrm{~nm}$ ) was applied from the window to generate carriers, instead of a voltage pulse as in DLTS measurements. Because of the internal electric field, minority carriers (electrons) will diffuse into the depletion region and fill the minority carrier traps. The majority excess holes will be swept out of the depletion region. The separation of excess electrons and holes will create a photovoltage on the Schottky junction and narrow down the depletion region. After the light pulse is ended, electrons start to emit out of the defect states, generating an exponential transient signal. When the voltage is biased at -2 $\mathrm{V}$, only a large peak at $38.8 \mathrm{~K}$ is observed (black curve in Figure $5 b$ ) due to the freezing-out of boron dopants. As the bias voltage is tuned to $-1 \mathrm{~V}$ (red curve in Figure $5 \mathrm{~b}$ ) and $-0.2 \mathrm{~V}$ (blue curve in Figure $5 \mathrm{~b}$ ), several spectral features show up. First, the $38.8 \mathrm{~K}$ peak amplitude increases as the bias voltage increases, because the detection region moves toward the surface where the boron concentration is higher. Second, a shoulder peak appears on the right side of the spectral peak, which likely comes from several closely spaced peaks related to minority traps. It is difficult to identify the peaks buried in the shoulder by the Arrehnius plot. But the MCTS simulations (Supplementary Note 5) help us identify them as carbonrelated minority carrier traps. The defects of $\mathrm{C}_{\mathrm{s}} \mathrm{OH}(0.11$ and $0.13 \mathrm{eV})$ and $\mathrm{C}_{s} \mathrm{H}(0.15 \mathrm{eV})$ are located in the broad shoulder (Figure $5 \mathrm{~b}$, inset). Therefore, we conclude that carbon-related minority carrier traps of $\mathrm{C}_{s} \mathrm{H}$ and $\mathrm{C}_{\mathrm{s}} \mathrm{OH}$ are formed in Bdoped silicon by SAMM. The carbon depth profile and probing region simulations based on SIMS and CV data confirmed that these carbon-related minority carrier traps were generated during the SAMM doping (Figure 5c). When the bias voltage varies from -2 to $-0.2 \mathrm{~V}$, the detection region shifts from $\sim 631 \mathrm{~nm}$ (Figure 5e) to $\sim 29 \mathrm{~nm}$ (Figure 5d). Clearly, carbon-related defects appear only in the doping region. The concentration of these defect states is only on a few percent in comparison with the boron doping concentration.

The downscaling of modern (CMOS) transistors requires a precise doping technique at the nano- and atomic scale without lattice damage. Solution-based SAMM doping is able to meet all the challenges with the advantages of ultrashallow junction formation, conformal doping on 3D surfaces, no lattice damage, atomic precision, and mass production. However, the contamination of carbon that deactivates phosphorus dopants in silicon has been a major concern for the molecular monolayer doping technique. ${ }^{14,16,29,32}$ It is quite a challenge to avoid the impact of carbon contamination, although many approaches to increase the electrical activities of phosphorus dopants have been developed, such as breaking chemical bonds to release carbon at low temperature ${ }^{32}$ and low temperature annealing to transform interstitial carbon into substitutional carbon. ${ }^{29}$ The fact that carbon contaminants do not bind with boron dopants implies that high-performance deep nanoscale or even atomic-scale electronic or quantum devices may be readily developed using self-assembled molecular monolayers that carry boron dopants.

\section{CONCLUSION}

Boron dopants have been successfully introduced into silicon by self-assembled ABAPE monolayers. Unlike P-doped silicon, carbon from the organic molecules does not form complexities with boron dopants. Oxygen from the $\mathrm{SiO}_{2}$ layer can diffuse into the silicon substrate and form defects in the form of $\mathrm{B}_{\mathrm{s}}-$ $\mathrm{O}_{2 \mathrm{i}}$ by binding with a few percent of the boron dopants. Carbon from organic molecules can help suppress the concentration of $\mathrm{B}_{\mathrm{s}}-\mathrm{O}_{2 \mathrm{i}}$ defects. Carbon-related defects in form of $\mathrm{C}_{\mathrm{s}} \mathrm{H}$ and $\mathrm{C}_{\mathrm{s}} \mathrm{OH}$ were also detected as minority carrier traps, which are located in upper half of the band gap and have nearly no influence on boron activation rate. As a whole, boron reaches nearly full activation in SAMM-doped silicon.

\section{ASSOCIATED CONTENT}

\section{Supporting Information}

The Supporting Information is available free of charge at https://pubs.acs.org/doi/10.1021/acsaelm.9b00748.

Surface functionalization; angle-resolved XPS; van der Pauw measurements; calculations about active boron distribution; band structure simulation; MCTS simulation (PDF) 


\section{AUTHOR INFORMATION}

\section{Corresponding Author}

*E-mail: yaping.dan@sjtu.edu.cn.

\section{ORCID 웅}

Yaping Dan: 0000-0002-2983-7213

\section{Present Addresses}

${ }^{\#}$ Future Industries Institute, University of South Australia, Mawson Lakes 5095, SA, Australia (B.G.).

"School of Electrical and Electronic Engineering, Nanyang Technological University, 50 Nanyang Avenue, 639798, Singapore (X.G.).

\section{Notes}

The authors declare no competing financial interest.

\section{ACKNOWLEDGMENTS}

This work was financially supported by the National Science Foundation of China (61874072) and the Major Research Plan, Science and Technology Commission of Shanghai Municipality (16JC1400405). The authors appreciate Dr. Limin Sun and Ligang Zhou at the Instrumental Analysis Center (IAC), Shanghai Jiao Tong University, for XPS and Hall measurements.

\section{REFERENCES}

(1) Ho, J. C.; Yerushalmi, R.; Jacobson, Z. A.; Fan, Z.; Alley, R. L.; Javey, A. Controlled nanoscale doping of semiconductors via molecular monolayers. Nat. Mater. 2008, 7 (1), 62-67.

(2) Hersam, M. C.; Guisinger, N. P.; Lyding, J. W. Silicon-based molecular nanotechnology. Nanotechnology 2000, 11 (2), 70-76.

(3) Schofield, S. R.; Curson, N. J.; Simmons, M. Y.; Rueß, F. J.; Hallam, T.; Oberbeck, L.; Clark, R. G. Atomically precise placement of single dopants in Si. Phys. Rev. Lett. 2003, 91 (13), 136104.

(4) Thissen, P.; Fuchs, E.; Roodenko, K.; Peixoto, T.; Batchelor, B.; Smith, D.; Schmidt, W. G.; Chabal, Y. Nanopatterning on Hterminated $\mathrm{Si}(111)$ explained as dynamical equilibrium of the chemical reaction with methanol. J. Phys. Chem. C 2015, 119 (29), 16947-16953.

(5) Ye, L.; de Jong, M. P.; Kudernac, T.; van der Wiel, W. G.; Huskens, J. Doping of semiconductors by molecular monolayers: monolayer formation, dopant diffusion and applications. Mater. Sci. Semicond. Process. 2017, 62, 128-134.

(6) Ruess, F. J.; Oberbeck, L.; Simmons, M. Y.; Goh, K. E. J.; Hamilton, A. R.; Hallam, T.; Schofield, S. R.; Curson, N. J.; Clark, R. G. Toward atomic-scale device fabrication in silicon using scanning probe microscopy. Nano Lett. 2004, 4 (10), 1969-1973.

(7) McKibbin, S.; Polley, C.; Scappucci, G.; Keizer, J.; Simmons, M. Low resistivity, super-saturation phosphorus-in-silicon monolayer doping. Appl. Phys. Lett. 2014, 104 (12), 123502.

(8) Simmons, M. A single atom transistor. 2012 IEEE Silicon Nanoelectronics Workshop (SNW) 2012, 1-1.

(9) Guan, B.; Siampour, H.; Fan, Z.; Wang, S.; Kong, X. Y.; Mesli, A.; Zhang, J.; Dan, Y. Nanoscale nitrogen doping in silicon by selfassembled monolayers. Sci. Rep. 2015, 5, 12641.

(10) Ho, J. C.; Yerushalmi, R.; Smith, G.; Majhi, P.; Bennett, J.; Halim, J.; Faifer, V. N.; Javey, A. Wafer-scale, sub-5 nm junction formation by monolayer doping and conventional spike annealing. Nano Lett. 2009, 9 (2), 725-730.

(11) Ang, K.-W.; Barnett, J.; Loh, W.-Y.; Huang, J.; Min, B.-G.; Hung, P.; Ok, I.; Yum, J.; Bersuker, G.; Rodgers, M. 300mm FinFET results utilizing conformal, damage free, ultra shallow junctions $\left(\mathrm{X}_{j} \sim\right.$ $5 \mathrm{~nm})$ formed with molecular monolayer doping technique. 2011 IEEE International Electron Devices Meeting (IEDM) 2011, 35.5.135.5.4.
(12) Voorthuijzen, W.; Yilmaz, M. D.; Naber, W. J.; Huskens, J.; van der Wiel, W. G. Local doping of silicon using nanoimprint lithography and molecular monolayers. Adv. Mater. 2011, 23 (11), 1346-1350.

(13) Popere, B. C.; Russ, B.; Heitsch, A. T.; Trefonas, P.; Segalman, R. A. Large-area, nanometer-scale discrete doping of semiconductors via block copolymer self-assembly. Adv. Mater. Interfaces 2015, 2 (18), 1500421.

(14) Wu, H.; Guan, B.; Sun, Y.; Zhu, Y.; Dan, Y. Controlled doping by self-assembled dendrimer-like macromolecules. Sci. Rep. 2017, 7, 41299.

(15) Gao, X.; Guan, B.; Mesli, A.; Chen, K.; Dan, Y. Deep level transient spectroscopic investigation of phosphorus-doped silicon by self-assembled molecular monolayers. Nat. Commun. 2018, 9 (1), 118.

(16) Shimizu, Y.; Takamizawa, H.; Inoue, K.; Yano, F.; Nagai, Y.; Lamagna, L.; Mazzeo, G.; Perego, M.; Prati, E. Behavior of phosphorous and contaminants from molecular doping combined with a conventional spike annealing method. Nanoscale 2014, 6 (2), 706-710.

(17) Caccamo, S.; Puglisi, R. A.; Di Franco, S.; D’Urso, L.; Indelicato, V.; Italia, M.; Pannitteri, S.; La Magna, A. Silicon doped by molecular doping technique: role of the surface layers of doped $\mathrm{Si}$ on the electrical characteristics. Mater. Sci. Semicond. Process. 2016, 42, 200-203.

(18) $\mathrm{Wu}, \mathrm{H} . ; \mathrm{Li}, \mathrm{K}$; Gao, X.; Dan, Y. Phosphorus ionization in silicon doped by self-assembled macromolecular monolayers. AIP Adv. 2017, 7 (10), 105310.

(19) Taheri, P.; Fahad, H. M.; Tosun, M.; Hettick, M.; Kiriya, D.; Chen, K.; Javey, A. Nanoscale junction formation by gas-phase monolayer doping. ACS Appl. Mater. Interfaces 2017, 9 (24), 20648 20655.

(20) van Druenen, M.; Collins, G.; Glynn, C.; O’Dwyer, C.; Holmes, J. D. Functionalization of $\mathrm{SiO} 2$ surfaces for $\mathrm{Si}$ monolayer doping with minimal carbon contamination. ACS Appl. Mater. Interfaces 2018, 10 (2), 2191-2201.

(21) Haddara, Y. M.; Folmer, B. T.; Law, M. E.; Buyuklimanli, T. Accurate measurements of the intrinsic diffusivities of boron and phosphorus in silicon. Appl. Phys. Lett. 2000, 77 (13), 1976-1978.

(22) Altermatt, P. P.; Schenk, A.; Schmithüsen, B.; Heiser, G. A simulation model for the density of states and for incomplete ionization in crystalline silicon. II. Investigation of $\mathrm{Si}: \mathrm{As}$ and $\mathrm{Si}: \mathrm{B}$ and usage in device simulation. J. Appl. Phys. 2006, 100 (11), 113715.

(23) Schenk, A.; Altermatt, P. P.; Schmithusen, B. Physical model of incomplete ionization for silicon device simulation. 2006 International Conference on Simulation of Semiconductor Processes and Devices 2006, $51-54$.

(24) Lin, J. F.; Li, S. S.; Linares, L. C.; Teng, K. W. Theoretical analysis of hall factor and hall mobility in p-type silicon. Solid-State Electron. 1981, 24 (9), 827-833.

(25) Adey, J.; Jones, R.; Palmer, D. W.; Briddon, P. R.; Öberg, S. Degradation of boron-doped Czochralski-grown silicon solar cells. Phys. Rev. Lett. 2004, 93 (5), 055504.

(26) Schmidt, J.; Bothe, K. Structure and transformation of the metastable boron- and oxygen-related defect center in crystalline silicon. Phys. Rev. B: Condens. Matter Mater. Phys. 2004, 69 (2), 024107.

(27) Monakhov, E. V.; Nylandsted Larsen, A.; Kringho/j, P. Electronic defect levels in relaxed, epitaxial p-type $S_{1-x} \mathrm{Ge}_{\mathrm{x}}$ layers produced by $\mathrm{MeV}$ proton irradiation. J. Appl. Phys. 1997, 81 (3), $1180-1183$.

(28) Drevinsky, P. J.; Caefer, C. E.; Tobin, S. P.; Mikkelsen, J. C.; Kimerling, L. C. Influence of oxygen and boron on defect production in irradiated silicon. MRS Proceedings 1987, 104, 167-172.

(29) Devine, R. A. B. SiO2/Si Interfacial degradation and the role of oxygen interstitials. J. Phys. III 1996, 6 (12), 1569-1594.

(30) Gao, X.; Guan, B.; Mesli, A.; Chen, K.; Sun, L.; Dan, Y. Toward defect-free doping by self-assembled molecular monolayers: the evolution of interstitial carbon-related defects in phosphorus-doped silicon. ACS Omega 2019, 4 (2), 3539-3545. 
(31) Yoneta, M.; Kamiura, Y.; Hashimoto, F. Chemical etchinginduced defects in phosphorus-doped silicon. J. Appl. Phys. 1991, 70 (3), 1295-1308.

(32) Tokuda, Y.; Katoh, I.; Ohshima, H.; Hattori, T. Observation of hydrogen in commercial Czochralski-grown silicon wafers. Semicond. Sci. Technol. 1994, 9 (9), 1733-1735. 